\title{
Vorgehen bei suspektem und positivem Abstrich der Cervix uteri
}

\author{
Replik zum Kommentar zur Guideline [1]
}

\section{S. Heinzl für die Arbeitsgruppe «Guideline Zervixabstrich» der Qualitätssicherungskommission der SGGG}

Korrespondenz:

Prof. Dr. med. Siegfried Heinzl Frauenklinik

Kantonsspital Bruderholz

CH-4101 Bruderholz

E-Mail: siegfried.heinzl@ksbh.ch
Die Herren R. Rondez, J. Kunz und C. Moll haben einen kritischen Kommentar zur Guideline «Vorgehen bei suspektem und positivem zytologischen Abstrich der Cervix uteri» geschrieben [2]. Die Arbeitsgruppe «Guideline Zervixabstrich» der Qualitätssicherungskommission der SGGG möchte dazu eine klärende Replik abgeben.

Vorauszuschicken ist, dass eine Guideline nur allgemein anerkannte und erprobte Methoden in Diagnostik und Therapie empfehlen darf. Methoden, welche noch nicht abschliessend beurteilt, oder von vielen wichtigen Experten abgelehnt werden, können in Guidelines nicht Eingang finden.

Die Kommission hat einen solchen Kommentar erwartet, da zum einen von aussen immer wieder versucht wurde, auf die Arbeit Einfluss zu nehmen, zum anderen ist zu bemängeln, dass die Beendigung der Arbeit und die Publikation zu weit auseinanderliegen. Die Guideline «Vorgehen bei suspektem und positivem Abstrich der Cervix uteri» berücksichtigt die Literatur bis Oktober 2000 und wurde, nach der Verabschiedung durch die Kommission im Sommer 2001, erst im Januar 2003 publiziert [1]. Dies ist sicherlich ein gravierender Nachteil, da ja gerade in diesem speziellen Bereich alles im Fluss ist. Aus diesem Grunde wurde auch ein Nachtrag verfasst, welcher zwei wesentliche Arbeiten welche das Vorgehen verändern - berücksichtigt. Diese Gegebenheiten müssen aber bei einer kritischen Beurteilung berücksichtigt werden.

Nun zu den zwei Hauptkritikpunkten, nämlich die Nicht-Empfehlung der Dünnschichtmethoden und der HPV-Typisierung.

\section{Dünnschichtmethoden}

Dünnschichtmethoden sind nach wie vor stark umstritten und stehen, im Gegensatz zur Behauptung der Autoren, sehr wohl zur Diskussion. Warum hat die eidgenössische Leistungskommission des BSV die Dünnschichtzytologie - nachdem sie zweieinhalb Jahre befristet zulässig war - von der Liste gestrichen und die
Anwendung als nicht mehr kassenpflichtig erklärt [3]? Auch ist in den kürzlich adaptierten Richtlinien der «United States Preventive Services Task Force» (USPSTF) folgendes nachzulesen: «The USPSTF conclude that the evidence is insufficient to recommend for or against new technologies in place of conventional PAP tests.» [4]. Die Autoren des Kommentars haben einen nicht unwesentlichen Teil der Literatur, wie auch die Stellungnahmen z.B. der Deutschen Gesellschaft für Zytologie [5], des australischen «Medical Services Advisory Committee» [6] und Publikationen von wichtigen Exponenten - z. B. Coste J., Sawaya G.F, Schneider V. [7-10] u.a. - einfach ausgeblendet. So einfach sollte man es sich nicht machen. Auch der Hinweis, dass etwa 60 bis 70\% der Schweizer Gynäkologen und Gynäkologinnen die Dünnschichtzytologie anwenden, ist weniger ein Zeichen der Evidenz als viel mehr des gekonnten Marketings.

\section{HPV-Typisierung}

Die Bedeutung der HP-Viren für die Entstehung des Zervixkarzinoms ist allgemein anerkannt und daher unstrittig. Die Guideline behandelt aber das Vorgehen bei Vorliegen eines suspekten oder positiven Zytologiebefundes. Im Jahre 2000 standen die von Herren Rondez; Kunz und Moll angeführten Indikationen sehr wohl zur Diskussion, waren aber sicherlich noch nicht allgemein anerkannt. Im Nachtrag haben wir auf die mittlerweile nach wie vor eingeschränkte Empfehlung der High-risk-HPV-Typisierung hingewiesen. Dies muss den Autoren beim Studium der Guideline wahrscheinlich entgangen sein, da sie die bereits in unserem Nachtrag erwähnten Ergebnisse der Konsensuskonferenz nochmals zitieren. Nichts desto trotz möchte die Kommission - ausser den wenigen Fällen von persistierenden ASC-H, persistierender CIN I/ low grade SIL (über zwei Jahre) und Status nach Konisation mit Restbefunden - den Highrisk-HPV-Nachweis momentan nicht generell empfehlen [11]. Die Empfehlung eines Testes ist 
nur sinnvoll, wenn man je nach Testergebnis das Vorgehen relevant ändert. Dies ist bis heute noch nicht der Fall. Auch treten in der täglichen Praxis sehr viele Probleme auf, da immer noch sehr viel Falsches über die HPV-Infektion und die Testung verbreitet wird.

Hingegen ist jegliche Art der Forschung in diesem Bereich zu begrüssen und $\mathrm{zu}$ fördern, damit die hier aufgeworfenen Fragen baldmöglichst eindeutig beantwortet werden können.

Abschliessend ist festzuhalten, dass eine Guideline, so einfach wie möglich, das unbedingt Notwendige und Nützliche beinhalten sollte. Die Empfehlungen müssen nicht das maximale, sondern das vernünftige und wirtschaftlich Vertretbare enthalten. Eine Guideline stellt immer, nach Wertung der vorliegenden Daten, einen Kompromiss unter den Kommissionsteilnehmern dar. Wichtig ist aber, dass die Kommission unabhängig, frei von Einflüssen der Hersteller irgendwelcher Methoden, agiert.

Die Kommission hat, wie bereits im Nachtrag festgehalten, mit der Vorarbeit zur Revision begonnen. Sie wird alles daran setzen, dass die überarbeitete Version dann schneller publiziert wird, damit auch die Aktualität in allen Bereichen gegeben ist. Wichtig ist aber, dass die momentan vorliegende Guideline nach Beachtung des Nachtrages ihre volle Gültigkeit hat und somit als Richtschnur dienen kann. Schliesslich sollte man die hier aufgeworfenen Detailfragen nicht überbewerten, da das Problem der Bekämpfung des Zervixkarzinoms in der ungenügenden Teilnahme der Frauen am Screening und nicht im Vorgehen beim suspekten oder positiven Zytologiebefund liegt. In der Schweiz findet bekannterweise lediglich ein sogenanntes opportunistisches Screening statt. Dies bedeutet, dass viele «Risikofrauen» dem Screening fernbleiben. Diese Frage wird uns in Zukunft, bei allen anderen wichtigen Detailfragen, viel mehr zu beschäftigen haben.

\section{Literatur}

1 Arbeitsgruppe «Guideline Zervixabstrich». Guideline zum Vorgehen bei suspektem und positivem zytologischen Abstrich der Cervix uteri. Schweiz Ärztezeitung 2003;84(3):82-92.

2 Rondez R, Kunz J, Moll C. Kommentar zur Guideline zum Vorgehen bei suspektem und positivem zytologischen Abstrich der Cervix uteri. Schweiz Ärztezeitung 2003;84(23):1243-4.

3 Verordnung des EDI vom 29. September 1995 über Leistungen in der obligatorischen Krankenpflegeversicherung (Krankenpflege-Leistungsverordnung, KLV) EDI vom 29. September 1995 (Stand am 24. Dezember 2002), Anhang 1, Kapitel 3: Gynäkologie, Geburtshilfe. http://www.admin.ch/ch/d/sr/c832_112_31.html.

4 Agency for Healthcare Research and Quality. Screening for Cervical Cancer (Systematic evidence review from the U.S. Preventive Services Task Force [USPSTF]). AHRQ Publication No. APPIP03 - 0004, January 2003.

5 Freudenberg N. Stellungnahme der Deutschen Gesellschaft für Zytologie (DGZ) zum Einsatz der Dünnschichtzytologie. Gynäkologe 2003;32:257.

6 Medical Services Advisory Committee (MSAC). Liquid based cytology for cervical screening, reference 12a, Assessment report. MSCA Publications approval No. 3174. Commonwealth of Australia 2003. Dep. of Health and Ageing, Canberra, ACT, 2601; März 2003, ISBN Nr. 0642821771.

7 Coste J, Cochand-Priolett B, de Cremoux P, et al. Cross sectional study of conventional cervical smear, monolayer cytology, and human papillomavirus DNA testing for cervical cancer screening. Br Med J 2003;326:1-5.

8 Muth C, Valesco-Garrido M, Schneider V. Dünnschicht-Zytologie: Rechtfertigt die Evidenzlage einen breiten Einsatz? Frauenarzt 2003; 44(4):413-8.

9 Schneider V. Evaluation of liquid-based cytology in cervical screening of high-risk population. Cytopathology 2003;14:1.

10 Sawaya GF, Brown AD, Washington AE, et al. Current Approaches to Cervical cancer Screening. N Engl J Med 2001;344(21):1603-7.

11 Miller AB. The natural history of human papillomavirus infection of the cervix uteri relevance to screening and clinical management. Frauenarzt 2002;42:687-90 (deutsche Übersetzung). 